\title{
Endpoint of $r p$ process using relativistic mean field approach and a new mass formula
}

\author{
Chirashree Lahiri ${ }^{1}$ and G. Gangopadhyay ${ }^{2}$ \\ Department of Physics, University of Calcutta \\ 92, Acharya Prafulla Chandra Road, Kolkata-700 009, India \\ email: ${ }^{1}$ chirashree.lahiri@gmail.com, ${ }^{2}$ ggphy@caluniv.ac.in
}

May 25, 2018

\begin{abstract}
Densities from relativistic mean field calculations are applied to construct the optical potential and, hence calculate the endpoint of the rapid proton capture $(r p)$ process. Mass values are taken from a new phenomenological mass formula. Endpoints are calculated for different temperaturedensity profiles of various X-ray bursters. We find that the $r p$ process can produce significant quantities of nuclei upto around mass 95. Our results differ from existing works to some extent.
\end{abstract}

\section{Introduction}

Proton capture reactions at a very low temperature play an important role in nucleosynthesis process. Most importantly, in explosive nucleosynthesis (e.g. an X-ray burst), the rapid proton capture $(r p)$ process is responsible for the production of proton-rich isotopes upto mass 100 region. In nature, the proton capture reactions, important for nucleosynthesis, usually involve certain nuclei as targets which are not available on earth or cannot be produced in terrestrial laboratories with our present day technology. Therefore, theory remains the sole guide to extract the physics.

In our present work, we have studied the endpoint of the $r p$ process in a microscopic approach using a new phenomenological mass formula [1]. In a similar work, Schatz et al. [2] calculated the endpoint of $r p$ process using mass values from a Finite Range droplet Model (FRDM) 3 calculation and proton capture rates from Hauser-Feshbach code NON-SMOKER 4, 5]. We will show that the results of our calculation are different from their observations. In the present work, we will concentrate only on X-ray burst scenarios, which have typical timescale of 100 seconds and a peak proton flux density of the order of $10^{6} \mathrm{gram} / \mathrm{cm}^{3}$. This type of burst provides a highly proton-rich environment around the peak temperatures 1-2 GK. We try to look at different models of the X-ray burster and find out the endpoint of the $r p$ process nucleosynthesis. 


\section{Methodology}

When an X-ray burst takes place, a proton-rich high temperature environment, which triggers the $r p$ process, is created. The process passes through nuclei near the proton drip line, not available on earth. In regions far from the stability valley, rates derived from phenomenological calculations may not represent the reality very well, leading to considerable uncertainty in the process. Very often, the reaction rates are varied by a large factor to study their effects. On the other hand, in a microscopic calculation, uncertainty in reaction rates can be reduced and therefore, this approach is expected to give a more accurate result for unknown mass regions. In a previous work $[\underline{6}$, we have shown that the rates may vary at most by a factor less than two when the cross-section values range over four orders of magnitude.

A microscopic calculation has been performed to evaluate proton capture rates for the nuclei involve in the $r p$ process in the present work. We use the spherical optical model to calculate the rates of the relevant reactions. As most of the nuclei involved in the process lie around the drip line, experimental density information are not available. Hence, theoretical density profiles have been calculated using relativistic mean field (RMF) theory. In the present work, we use the FSU Gold Lagrangian density 7$]$ and solve the RMF equations in the spherical approximation for the nuclei involved in the $r p$ process. This Lagrangian density, containing additional nonlinear terms for the vector isoscalar meson self interaction and an interaction between the isoscalar vector and the isovector vector mesons, has been found to be very useful in describing nuclear properties throughout the mass table [See e.g. Bhattacharya et al[ 8 , 9] and references therein].

The microscopic optical model potential for capture reactions are obtained using effective interactions derived from the nuclear matter calculation in local density approximation, i.e. by substituting the nuclear matter density with the density distribution of the finite nucleus. In the present work, we have constructed the potential by folding the density dependent M3Y (DDM3Y) 10, 11, 12 interaction with densities from RMF approach. This interaction was extracted from a finite-range energy-independent $G$-matrix element of the Reid potential by adding a zero-range energy-dependent pseudo-potential and a densitydependent factor. The interaction at the point $\vec{r}$ is, thus, given by

$$
v(r, \rho, E)=t^{M 3 Y}(r, E) g(\rho)
$$

where $E$ is the incident energy and $\rho$, the nuclear density. The $t^{M 3 Y}$ interaction is given by

$$
t^{M 3 Y}=7999 \frac{e^{-4 r}}{4 r}-2134 \frac{e^{-2.5 r}}{2.5 r}+J_{00}(E) \delta(r)
$$

for $r$ in $f m$, and $J_{00}(E)$ is the zero range pseudo potential,

$$
J_{00}(E)=-276\left(1-0.005 \frac{E}{A}\right) \mathrm{MeVfm}^{3}
$$

The density dependent factor $g(\rho)$ has been chosen of the form $C\left(1-\beta \rho^{2 / 3}\right)$ from the work by Chaudhuri 11, 12, where the constants were obtained from a nuclear matter calculation as $C=2.07$ and $\beta=1.624 \mathrm{fm}^{2}$. We have used this form in our calculation without changing any of the above parameters. 
We have also fixed the various parameters and prescriptions in the HauserFeshbach calculation for the relevant mass region by comparing our results to the experimental low energy proton capture cross sections for these nuclei. Our method of calculation for mean field and proton capture rates has been described in our earlier works [6, 13] in detail. The computer code TALYS 14] has been used for rate calculation.

Binding energy of nuclear ground state is one of the most important inputs in the study of astrophysical reactions. Experimental measurements are very difficult to perform in nuclei far from the stability line. Therefore, one has to take recourse to theoretical predictions. Though we have used a mean field calculation to extract the nuclear density profiles, no RMF calculation has been able to achieve a prediction of mass values with accuracy sufficient for studying the proton drip line. In fact, even Skyrme Hartree-Fock calculations can predict the mass values with an root mean square (rms) error slightly less than 0.6 $\mathrm{MeV}$ only. Thus, in the present work, we have obtained the mass values from a newly developed mass formula[1]. It uses a purely phenomenological form with empirically fitted parameters and predicts the known mass values of 2140 nuclei with an rms error of $0.376 \mathrm{MeV}$. In view of the success of the formula to predict the proton dripline and $r p$ process upto mass 80 region 15 and to predict the peaks in $r$ process [16] quite well, it will be interesting to see the effect of this mass formula on $r p$ process beyond mass 80 region and to the endpoint of the rp process.

In an X-ray burst environment, a nucleus $(Z, A)$ may capture a proton to form the nucleus $(Z+1, A+1)$. However, this process has to compete with its inverse, i.e. photodisintegration by emitting a proton at high temperature $[(\gamma, p)$ reaction]. A negative or a small positive value of the proton separation energy implies that the inverse reaction dominates and the $r p$ process stalls at that point, the so-called waiting point. Therefore, only a two proton capture process can bridge the waiting point nuclei. The bridging mechanism has been discussed in standard text books (for example the book by Illiadis [17]). For the $X(p, \gamma) Y$ reaction, the rate $\lambda$ of the inverse process $(\gamma, p)$ is related to the the proton capture rate by the reciprocity theorem and is of the form [17.

$$
\begin{array}{r}
\lambda=9.86851 \times 10^{9} T^{\frac{3}{2}}\left(\frac{M_{p} M_{X}}{M_{Y}}\right)^{\frac{3}{2}} \frac{\left(2 J_{p}+1\right)\left(2 J_{X}+1\right)}{\left(2 J_{Y}+1\right)} \frac{G_{p} G_{X}}{G_{Y}} \\
N\langle\sigma v\rangle_{p X \rightarrow Y \gamma} \exp \left(\frac{-11.605 Q}{T}\right)
\end{array}
$$

in the unit of $\sec ^{-1}$ for $X(p, \gamma) Y$ process. The forward reaction rate, denoted by $N\langle\sigma v\rangle_{p X \rightarrow Y \gamma}$, is expressed in $\mathrm{cm}^{3} \mathrm{~mol}^{-1} \mathrm{sec}^{-1}$. The temperature $T$ is in GK $\left(10^{9} \mathrm{~K}\right)$ and $Q$ is the ground state $\mathrm{Q}$-value of the $(p, \gamma)$ reaction expressed in $\mathrm{MeV}$. The normalized partition functions, $G_{X}$ and $G_{Y}$, have been obtained from Rauscher et al. [18] For protons, we use the standard values, $G_{p}=1$ and $J_{p}=1 / 2$. As evident from the above expression, the Q-value, appearing in the exponent, plays a vital role in the whole process.

Apart from the above processes, a nucleus can decay by emitting beta particles while, for higher mass isotopes, $\alpha$-decay is another probable channel. In this work, the measured half life values for $\beta$-decay have been taken from the compilation by Audi et al. 19] except in the case of ${ }^{65} \mathrm{As}$, which is taken from the experimental measurements by López et al.20 In absence of experimental 
data, half life values have been taken from the calculation by Möller et al. 3 both for $\beta$ - and $\alpha$ - decay. Taking into account all the above processes, we have constructed a network extended upto $A=110$ region to study the nucleosynthesis path and relative abundances of the elements at any instant.

\section{Results}

The $r p$ process paths are shown in Fig. 1 and Fig. 2 for temperatures 1.2 and $1.5 \mathrm{GK}$, respectively, using a constant proton flux density of $10^{6} \mathrm{gram} / \mathrm{cm}^{3}$, a proton fraction of 0.7 and 100 seconds burst duration. Here, black lines indicate the path along which the major portion of the total flux flow whereas gray lines indicate the minor paths. The filled boxes in figures indicate the waiting points. As evident from these figures, $r p$ process paths depend on the temperature of the environment to some extent. For example, at $\mathrm{T}=1.2 \mathrm{GK}$, major portions of the $r p$ process flux at the waiting point nucleus ${ }^{64} \mathrm{Ge}$ convert to ${ }^{66} \mathrm{Se}$ by twoproton capture process. Less than $1 \%$ of the total flux flows through the $\beta$-decay channel and follow the paths showed by gray lines in Fig. 1. In contrast, at temperature $\mathrm{T}=1.5 \mathrm{GK}$ (in Fig. 2), the probability of two-proton capture of ${ }^{64} \mathrm{Ge}$ gets reduced and the $r p$ process path bifurcates from the waiting point nucleus almost in an equal proportion. This suggests that, at $1.2 \mathrm{GK}$, proton capture by ${ }^{64} \mathrm{Ge}$ dominates over its inverse process, i.e. photodisintegration. As the temperature increases, contributions of photodisintegration process increases and therefore a large fraction of the total abundance chooses another path through more stable nuclei such as ${ }^{65} \mathrm{Ge},{ }^{66} \mathrm{As}$, etc. Similarly, near other waiting points (viz. ${ }^{72} \mathrm{Kr},{ }^{76} \mathrm{Sr}$ etc.) the abundance flow pattern changes with changing temperature. It is also evident from above the figures that, beyond mass 80 , temperature change can hardly affect the scenario.

As shown in Fig. 1 and Fig. 2, above $\mathrm{A}=100$ region, the $r p$ process continues through proton capture by In isotopes and $\beta$-decay of $\mathrm{Sn}$ isotopes. Here, ${ }^{100} \mathrm{In}$ captures a proton to form ${ }^{101} \mathrm{Sn}$ which completely decays to ${ }^{101} \mathrm{In}$ as ${ }^{102} \mathrm{Sb}$ is proton unbound. In turn, ${ }^{101}$ In undergoes proton capture and further exhibits $\beta$ decay. The process of proton capture followed by consecutive $\beta$-decay continues and relative abundances of nuclei decrease as one proceeds towards higher mass region. Ultimately, less than $0.001 \%$ of the total flux can reach ${ }^{106} \mathrm{In}$, according to our present calculation.

According to the calculations of Schatz et al. [2], a significant portion of the ${ }^{105} \mathrm{Sn}$ captures a proton to form ${ }^{106} \mathrm{Sb}$, as ${ }^{106} \mathrm{Sb}$ has a sufficient positive proton separation energy (0.59 MeV from FRDM[3] calculation). Another proton capture leads to ${ }^{107} \mathrm{Te}$, which instantly undergoes $\alpha$-decay to ${ }^{103} \mathrm{Sn}$. Thus, the $r p$ process ends in the SnSbTe cycle.

In contrast, our calculation does not go through the SnSbTe cycle. It is clear that the SnSbTe cycle may not occur under two scenarios. Firstly, if ${ }^{106} \mathrm{Sb}$ isotope be very loosely bound (proton separation energy $0.119 \mathrm{MeV}$ according to the mass formula[1]), any ${ }^{106} \mathrm{Sb}$ that is formed by a proton capture instantly reverts back to ${ }^{105} \mathrm{Sn}$. Second scenario occurs when the proton capture rates are too small for ${ }^{105} \mathrm{Sn}$ to initiate a proton capture process.

In a previous work 13, we have shown that a small fluctuation in the mass values of waiting point nuclei in mass 60-80 region may affect the effective half life and thus, proper knowledge of ground state binding energy is necessary to 
understand the bridging phenomena of a waiting point nucleus below mass 80 region. However, as we move towards the higher mass region, we find that small variations in binding energy do not affect the $r p$ process path significantly. Taking the rms error into account for the proton separation energy of ${ }^{106} \mathrm{Sb}$ $(0.119 \pm 0.376) \mathrm{MeV}$, we have checked whether the proton capture on ${ }^{105} \mathrm{Sn}$ can dominate over its inverse process. We find that the fraction of the initial flux entering into the SnSbTe-cycle is negligibly small. We have repeated our calculation with the proton separation energy of ${ }^{106} \mathrm{Sb}$ isotope from a recent experiment by Elomma et al. 21], viz. $0.428(0.008) \mathrm{MeV}$. We find that our results remain almost invariant. Repeating the entire calculation with ground state binding energies from FRDM 3 calculation or the Duflo-Zuker 22 mass formula do not alter this conclusion. Hence, we see that for a reasonable variation of mass values the $r p$ process fails to enter into the SnSbTe-cycle for an X-ray burst of 100 seconds duration. If we consider an X-ray burst having duration greater than 150 seconds, a very small fraction of total flux, less than $0.001 \%$ of the original, enters the cycle.

Schatz et al. 2 have used capture rates different from ours. In Fig. 3, we have plotted proton capture rates with temperature for the isotopes ${ }^{105} \mathrm{Sn}$ and ${ }^{106} \mathrm{Sb}$ respectively. The different values have been indicated as follows: Pres - Present work and NON-SMOKER - NON-SMOKER 4, 5] results used by Schatz et al. 2. In case of ${ }^{105} \mathrm{Sn}$ nuclei, it is evident from Fig. 3 that proton capture rates from our calculation are approximately $10^{2}$ times smaller than the rates from NON-SMOKER calculation. It seems, the difference is mainly due to the fact that they have used the form of the interaction from Jeukenne et al. 23] which is different from our case. For better understanding, we have repeated the entire calculation using reaction rates from Hauser-Feshbach code NON-SMOKER 4, 5]. In this case, considerable fraction of the initial flux $(\sim$ $0.1 \%$ ) enters into the SnSbTe cycle. Thus, we may conclude that the proton capture rates, and not the mass values, determine the endpoint of $r p$ process. In our opinion, our approach in fixing the parameters in the reaction calculation by fitting known reaction rates, and extrapolating the calculation to unknown reactions, may be relied upon to predict the endpoint correctly.

There are many X-ray burster models in literature with various densitytemperature profiles. Our present goal is to study how different temperaturedensity profiles can affect the relative abundances of the nuclei produced by $r p$ process and the endpoint. In Fig. 4, we have plotted the relative abundances of elements versus mass number at 100th second of the burst in various models, as described below.

In the first case (Model-I), we assume a constant density-temperature framework with temperature $T=1.5 \mathrm{GK}$, density $\rho=10^{6} \mathrm{gram} / \mathrm{cm}^{3}$ and proton fraction value 0.7. Model-II[2] describes a situation where ignition takes place at a constant density of $\rho=1.1 \times 10^{6} \mathrm{gram} / \mathrm{cm}^{3}$ and the burst reaches a peak temperature $T_{\text {peak }}=1.9 \mathrm{GK}$ after 4 seconds while the cooling phase lasts for approximately 200 seconds. In another example, taken from the book by Illiadis 17 (Model-III), nuclear burning starts with temperature and density values of $T=0.4 \mathrm{GK}$ and $\rho=10^{6} \mathrm{gram} / \mathrm{cm}^{3}$, respectively. After 4 seconds, a maximum temperature of $T_{\text {peak }}=1.36 \mathrm{GK}$ and a minimum density of $\rho_{\text {peak }}=5 \times 10^{5} \mathrm{gram} / \mathrm{cm}^{3}$ are achieved. After 100 seconds, the temperature drops to $T=0.7 \mathrm{GK}$ and the density increases to $\rho=1.4 \times 10^{6} \mathrm{gram} / \mathrm{cm}^{3}$. For all the above models, it is assumed that the X-ray burst environment is suffi- 
ciently proton-rich to maintain the number 0.7 as the constant proton fraction. As a significant amount of proton flux is consumed during the thermonuclear reaction, another situation may arise, where the proton fraction decreases gradually with time. Such a situation is given in Model-IV where the proton fraction decreases to 0.16 after 100 seconds with temperature-density profile same as that of the Model-III. It is evident from Fig. 4 that abundance peaks in all cases are obtained at mass values of 72,76 and 80 , as a result of the existence of waiting point nuclei ${ }^{72} \mathrm{Kr},{ }^{76} \mathrm{Sr}$ and ${ }^{80} \mathrm{Zr}$, respectively. Other peaks (for example, peaks at mass 85, 93 and 95 for isotopes ${ }^{85} \mathrm{Mo},{ }^{93} \mathrm{Pd},{ }^{95} \mathrm{Cd}$, respectively) suggest that the $r p$ process flux gets accumulated at these points due to very small positive or negative proton separation energies of those isotopes. We have considered the mass number in each case for which the flux amount drops below $1 \%$ of the initial flux. It is evident from Fig. 4 that for Model-I, $\mathrm{A} \approx 93$ is the region above which the $r p$ process flux fall below the range of our interest, whereas for Model-II, the region is around $\mathrm{A} \approx 95$. Other abundance peaks are observed at masses $A=97$ and 101 for the isotopes ${ }^{97} \mathrm{Cd}$ and ${ }^{101} \mathrm{Sn}$ respectively, though, the fractions of the total flux accumulated at those isotopes are less than $0.1 \%$ of the initial value. In case of Models III and IV, the locations to be studied are around $\mathrm{A} \approx 93$ and 91 respectively. From above observations, it can be concluded that, for various density-temperature profiles, the $r p$ process flux falls below a significant amount near mass 90-95. The observations from Fig. 4 suggest that the end points of the $r p$ process has a rather weak dependence on different X-ray burster models.

\section{Summary}

The location above which the $r p$ process flux falls below an insignificant amount is calculated using the microscopic optical model utilizing the densities from the RMF approach and with a new phenomenological mass formula. Present result is compared with result obtained from another existing work $[2]$ and the reason for these indifferences between the results are discussed. Our results do not significantly depend on the mass models. For different X-ray burster models, endpoints are calculated.

\section{Acknowledgments}

This work has been carried out with financial assistance of the UGC sponsored DRS Programme of the Department of Physics of the University of Calcutta. Chirashree Lahiri acknowledges the grant of a fellowship awarded by the UGC.

\section{References}

[1] G. Gangopadhyay, Int. J. Mod. Phys. E 20 (2011) 179.

[2] H. Schatz et al. Phys. Rev. Lett. 86 (2001) 3471.

[3] P. Möller, J. R. Nix, and K. L. Kratz, At. Data Nucl. Data. Tables 66 (1997) 131. 
[4] T. Rauscher and F.-K. Thielmann, At. Data Nucl. Data. Tables 75 (2000) 1.

[5] T. Rauscher and F.-K. Thielmann, At. Data Nucl. Data. Tables 76 (2001) 47.

[6] C. Lahiri and G. Gangopadhyay, Phys. Rev. C 84 (2011) 057601.

[7] B. G. Todd-Rutel and J. Piekarewicz, Phys. Rev. Lett. 95 (2005) 122501.

[8] M. Bhattacharya and G. Gangopadhyay, Phys. Rev. C 77 (2008) 047302.

[9] M. Bhattacharya, G. Gangopadhyay and S. Roy, Phys. Rev. C 85 (2012) 034312 .

[10] A.M. Kobos, B.A. Brown, R. Lindsay, G.R. and Satchler, Nucl. Phys. A 425 (1984) 205.

[11] A.K. Chaudhuri, Nucl. Phys. A 449 (1986) 243.

[12] A.K. Chaudhuri, Nucl. Phys. A 459 (1986) 417.

[13] C. Lahiri and G. Gangopadhyay, Eur. Phys. J. A 47 (2011) 87.

[14] A. J. Koning, S. Hilaire, and M. Duijvestijn, Proceedings of the International Conference on Nuclear Data for Science and Technology, April 2227, 2007, Nice, France, edited by O. Bersillon, F. Gunsing, E. Bauge, R. Jacqmin, and S. Leray (EDP Sciences, Paris, 2008), pp. 211214.

[15] C. Lahiri and G. Gangopadhyay, Int. J. Mod. Phys. E 20 (2011) 2417.

[16] C. Lahiri and G. Gangopadhyay, to appear in Int. J. Mod. Phys. E (2012).

[17] C. Illiadis, Nuclear Physics of the Stars (Wiley-VCH Verlag GmbH, Weinheim, 2007).

[18] T. Rauscher and F-K Thielemann, At. Data Nucl. Data. Tables 74 (2000) 1.

[19] G. Audi, O. Bersillon, J. Blachot and A.H. Wapstra, Nucl. Phys. A 729 (2003) 3 .

[20] M.J. López Jiménez et al., Phys. Rev. C 66 (2002) 025803.

[21] V.- V. Elomaa et al. Phys. Rev. Lett. 102 (2009) 252501.

[22] J. Duflo and A.P. Zuker, Phys. Rev. C 52 (1995) R23.

[23] J. Jeukenne, A. Lejeune, and C. Mahaux, Phys. Rev. C 16 (1977) 80. 


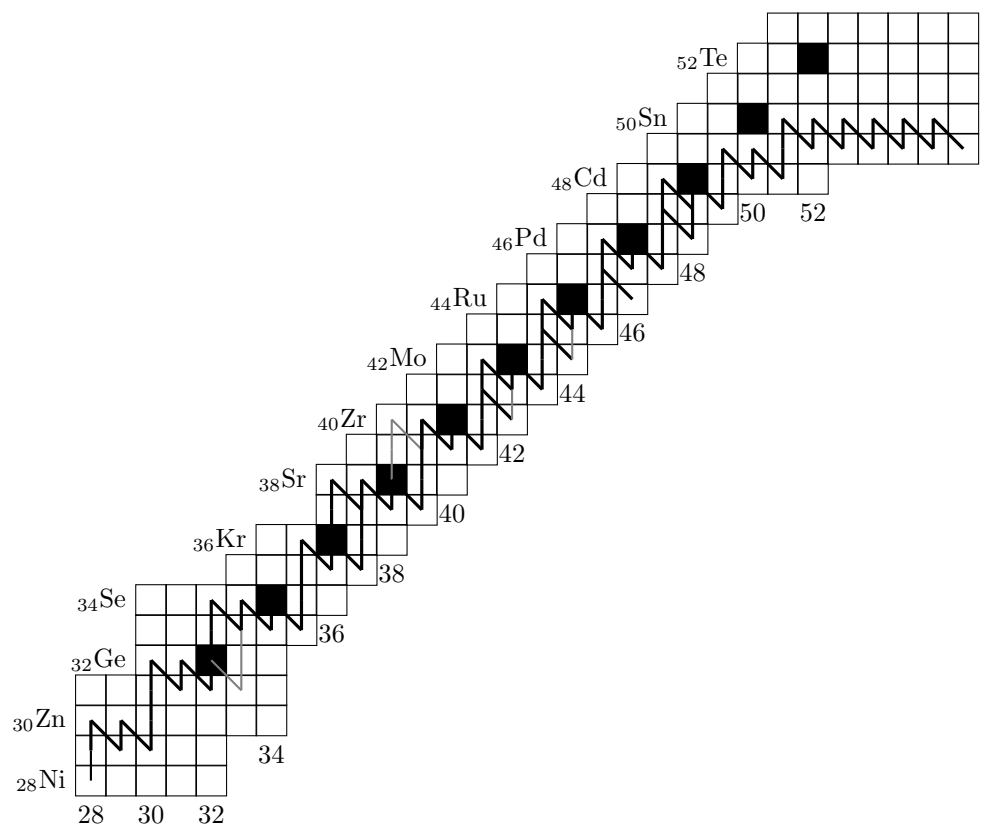

Figure 1: $r p$ process path for 1.2 GK

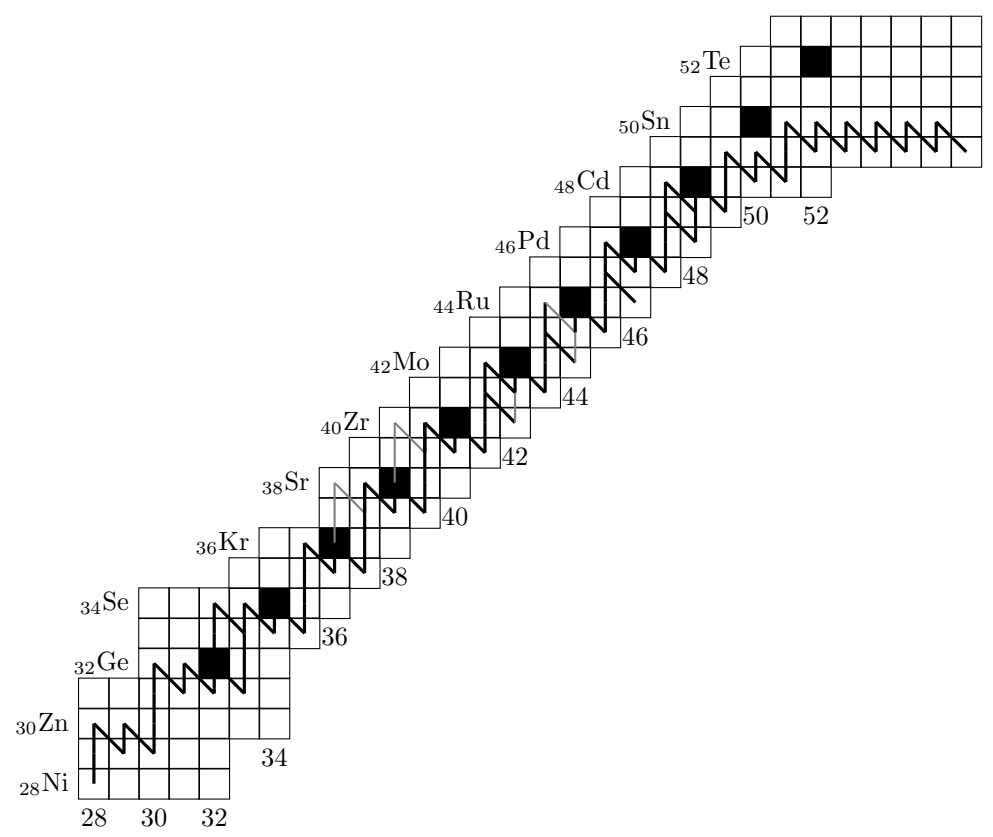

Figure 2: $r p$ process path for 1.5 GK 

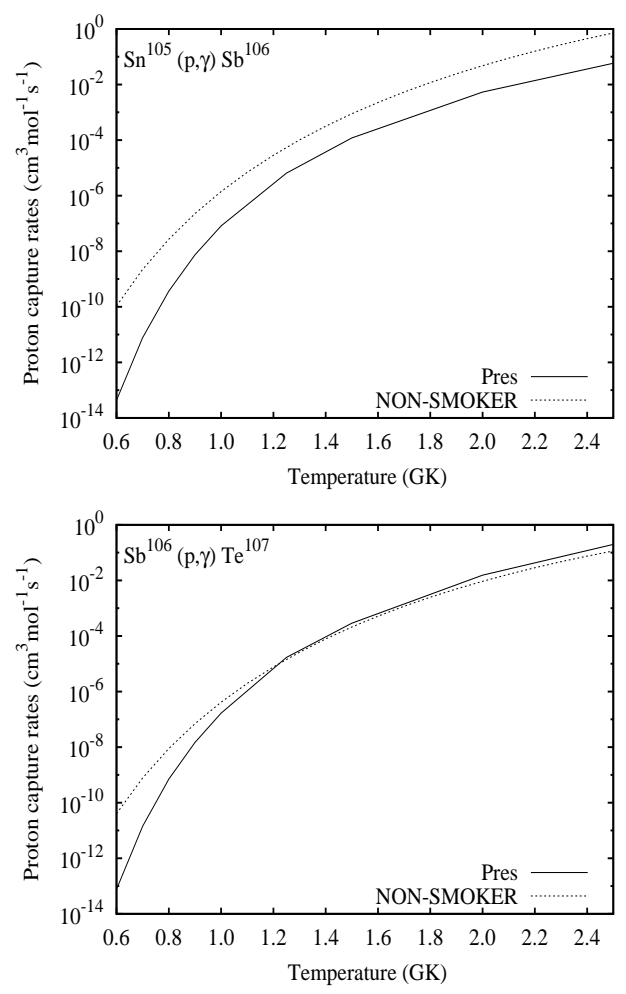

Figure 3: Proton capture rates $\left(\mathrm{cm}^{3} \mathrm{~mol}^{-1} \mathrm{sec}^{-1}\right)$ vs temperature(GK). See text for details. 

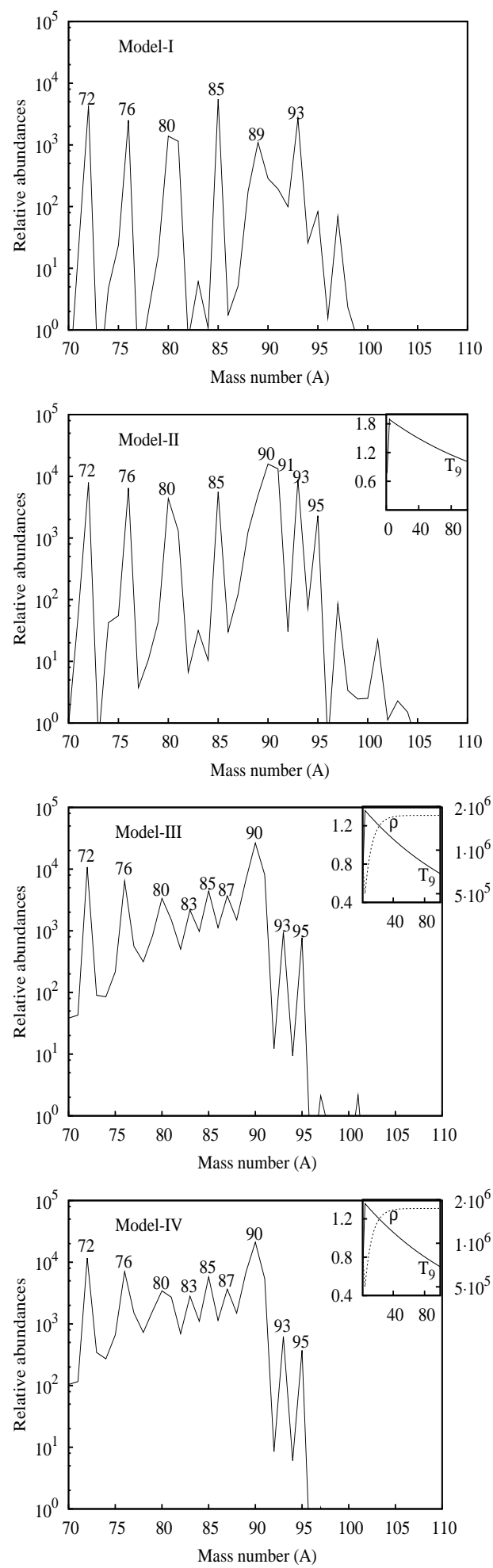

Figure 4: Relative abundances vs mass number for different density-temperature profiles of various X-ray burster after 100 seconds. (Insets) Model-II: Curve for temperature in GK vs time in seconds of the X-ray burst from Schatz et al. 2]; Model-III and IV: Temperature in GK (left) and density $\rho$ in $\mathrm{gram} / \mathrm{cm}^{3}$ (right) with time in seconds from Illiadis 17 . See text for details. 